\title{
The Homodimerization of Thalictrum tuberosum $O$-Methyltransferases by Homology-based Modelling
}

\author{
Heejung Yang, Joong-Hoon Ahn, Karp Joo Jeong, Sangsan Lee, and Yoongho Lim* \\ Bio Molecular Informatics Center, Konkuk Lnivensitv. Seoul 1+3-701, Korea \\ ${ }^{\dagger}$ Supercomputing Center, Korea Institute of Science and Techologi Information. Daejeon $305-350$. Korea \\ Received March 6, 2003
}

\begin{abstract}
Two $O$-methyltransferases. OMTII-I and OMTII-4 of meadow ne Thalictrum tuberostm showed a high sequence identity: Of 364 amino acids only one residue is not the same. which is Tyr2l or Cys 21 . Even if the 21 st residues in these OMTs are not included in the binding sites of the enzymes, binding affinities of the enzy me homodimers over the same substrate are very different. While the binding affinity of one homodimer over caffeic acid is $100 \%$. that of the other is $25 \%$. Authors tried to predict the three-dimensional structures of Thalictrum tuberosum $O$-methyltransferases using homology-based modelling by a contparison with caffeic acid $O$-methyltransferase, and explain the reason of the phenomenon mentioned above based on their three dimensional structural studies. In the enzyme homodimer, the better binding affinity may be caused by the shorter distance between the 21st residue and the binding site of the other monomer.
\end{abstract}

Key Words : Modelling. Molecular dynamics, Homology. Methyltransferase. Thalictrum tuberosum

\section{Introduction}

Since secondary metabolites of plants play an important role in improving disease resistance of plants against pathogens as well as promoting human health. an increasing number of research groups are studying their biosynthetic pathways.' Among many enzymes participated in their bioșythetic pathwayss. methyltransferases are ubiquitous and create more diverse class of metabolites. Secondary metabolites such as lignin. suberin. flavonoids. antocyanins. and isoflavonoids which are synthesized via phery lpropanoid pathway are also modified by methy ltransferases. ${ }^{2.3}$ Because they show diversity and play an important role in plant growth and development as well as the interactions of plants with their enrironment. many studies on methyltransferases have been carried out. ${ }^{+}$Especially, O-methỵltransferases (OMTs) are more common. OMTs can be classified based on their functions: (1) The first group methylates phenylpropanoids in the lignin biosynthesis pathway. (2) The second group methỵlates flavonoids. including chalcones. flavonols and flavones. (3) The third group methylates alkaloids and other chemicals such as sugars and scent compounds. ${ }^{5}$ In all three groups. OMTs transfer the methyl group of $S$-adenosyl-L-methionine (SAM) to the hydroxyl group of phenolic acceptors and form their methyl ether derivatives and $S$-adenosyl-L-homocysteine (SAH) as products. $^{6} O$-methylation not only reduces the chemical reactivity of their phenolic hydroxyl groups but also increases their lipophilicity and their intracellular compartmentation. $^{7}$ Therefore, to know the function of OMTs at a molecular level, many genes encoding OMTs have been isolated and are characterized. In contrast with mammalian

"Corresponding author. Tel: $+82-2-450-3760$; Fax: $+82-2-453-$ 3761: E-mail: yoongho takonkuk.ac.kr
OMTs. plant OMTs show narrow substrate specificities as well as position-specific activities. so that the homologybased comparison such as BLAST could not give enough information about the biological function of each OMTs such as substrate and product. ${ }^{8}$ Molecular modelling method may help us understand the reason why plant OMTs show narrow substrate specificities as well as position-specific activities. $^{9.10}$

It is known that methyl jasmonate can induce an alkaloid. berberine in cell cultures of meadow ne Thalictrim tuberosum. ${ }^{l l}$ Four cDNA encoding $O$-methyltransferases were isolated. Among these. two cDNAs named OMT II-I and OMT II-4 showed high sequence homology: Of 364 amino acids only one residue is not the same, which is Tyr2l of OMT II-1 and Cy's2l of OMT II-4. Even though both enzymes have the same substrate. caffeic acid, their binding affinities are different. The binding affinity values over caffeic acid of enzyme homodimers were reported by Frick and Kutchan. "l While in the case of OMT II-l homodimer the binding affinity over caffeic acid is $100 \%$. that of OMT II- 4 homodimer is $25 \%$. Of course. if the different amino acid is positioned at the binding site. this phenomenon may be explained. Compared with residues neighboring the binding site of caffeic acid $O$-methyltransferase (COMT) which shows a high homology over $74 \%$ with OMT II. their binding site can be predicted but the 21 st residue is not included there. ${ }^{8}$ Authors tried to predict the threedimensional (3D) structures of Thalictrum niberosum OMTs using homology-based modelling. A template for homology-based modelling was COMT deposited in Protein Data Bank (PDB code: 1KYZ.pdb). ${ }^{12}$ Based on their 3D structural studies, we tried to explain the reason why two enzyme homodimers show very different binding affinity over caffeic acid. 


\section{Materials and Methods}

Target and template proteins. The amino acid sequence of OM[ 1]-1 was obtained from GenBank (Accession No. Af064693: protein ID. AAD2984I). It is composed of 364 amino acids. The primary sequence of $\mathrm{OMJ}$ [I-4 was obtained from GenBank (Accession No. AF064696; protein ID. AAD29844) too. There is only one different residue between OMT II- 1 and OMT II-4, which is Tyr2I and Cys21, respectively. A template for homology-based modelling was COMT deposited in Protein Data Bank (PDB code: IKYZ. pdb). ${ }^{12}$ The number of amino acids of COMT is $365 . \mathrm{A}$ comparison of OMT 11-1. OMT II-4, and COMT is shown in Figure 1. 1KYZ.pdb includes three polymer chains such as A, C, and E. Among the three chains, the chains $\mathrm{A}$ and $\mathrm{C}$ consisted of a dimer and the chain $E$ exists as a monomer. Since homodimers are studied in this work, the chain $\mathrm{A}$ contained in COMT dimer was chosen as a template. The 3D structure of SAH produced from SAM by the enzyme was botrowed from $1 \mathrm{KYZ}$.pdb. The 3D structure of the substrate, cafteic acid was adopted from IKOU.pdb which is the complex of photoactive yellow protein and cafteic acid. ${ }^{1.3}$

Molecular modelling. The molecular modelling calculations were carried out on an O2 RI2,000 Silicon Graphics work-

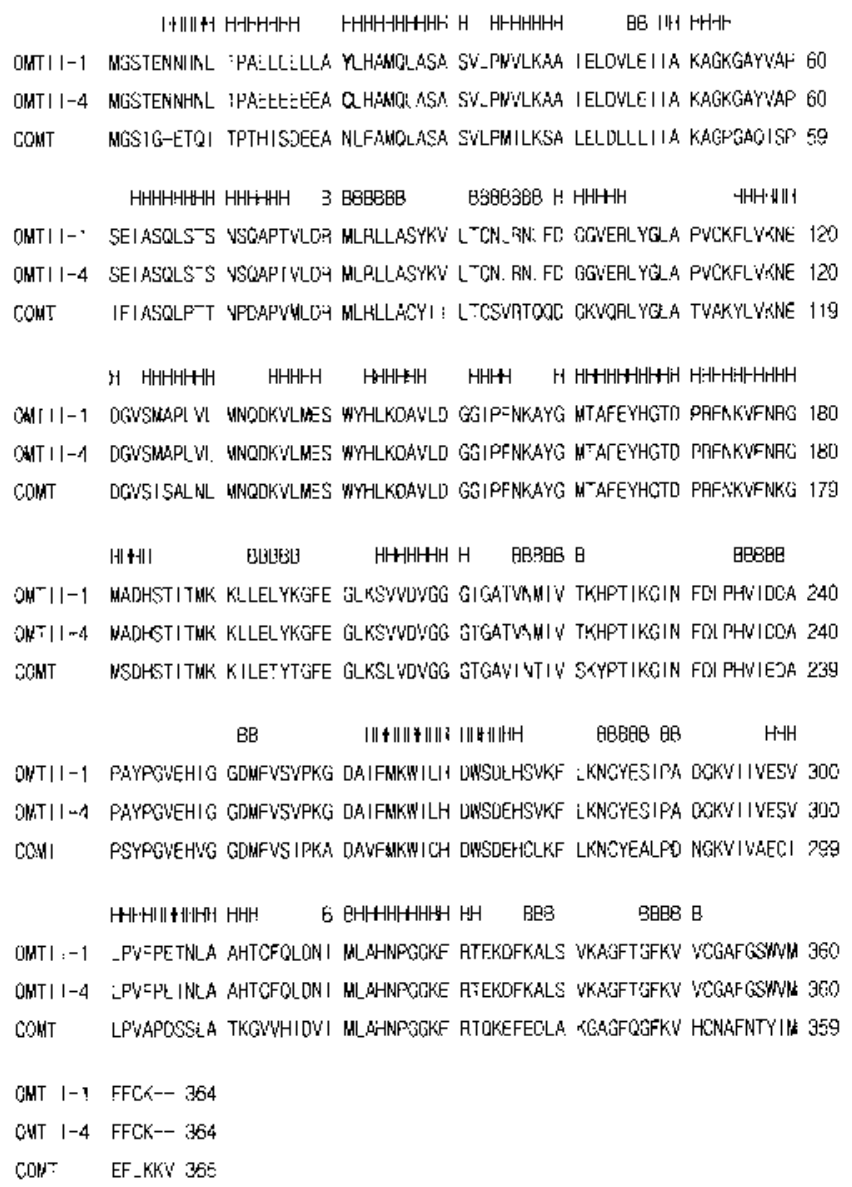

Figure I, A comparison of the primary secjuences of OM' II-I. OM'l' II-4. and COMl, (Bold characters denote Tyr2l of OMT II-1 and Cys2I of OMT II-4. H and B denote $\alpha$-helix and $\beta$-sheet. respectively.) station. The modelling software was Insightll (Accelrys, San Diego, USA.). The forcefield used for molecular dynamics (MD) and energy minimization was cuff provided by Accelrys. Homology module included in Insightll was used for homology modelling. ${ }^{4}$ the sequence of the template protein was extracted and aligned with the target protein. The structurally conserved regions (SCRs) were determined manually. With SCRs, the sequence was aligned. The loops and the variable regions not to be included in SCRs were built. The conformations of side chains were determined by conformational search of rotamers. The protein was embedded in a $5 \AA$ shell of 2,153 water molecules to imitate aqueous solvent conditions. The assembled molecules were subject to energy minimization by lnsightll/Discover module. Steepest descents were carried out until maximum derivative of $1.0 \mathrm{kcal} / \mathrm{mol} \AA$, and conjugate gradients were followed until maximum derivative of $0.1 \mathrm{kcal} / \mathrm{mol} \AA$. After energy minimization. MD was performed at $300 \mathrm{~K}$ for $1,000 \mathrm{psec}$ with 1 fsec each step. The output conformers were collected at every 4 psec. 250 conformers were saved in the history file. The energy profile was analyzed using InsightlL/Analysis module. For a statistical evaluation of the conformer, PROC'HECK was applied.

Docking of cafteic acid and SAH. The substrate, caffeic acid and the cofactor, SAH were docked into the protein using [nsightl]/Docking module on a Silicon Graphics $\mathrm{O}_{2}$ RI2,000 workstation. A ligand and protein assembly was created using a grid docking method. First, a grid was prepared surrounding the protein. After setup the grid, the ligand was docked. The ligand and protein assembly was soaked in a $5 \AA$ shell of 2,153 water molecules to imitate aqueous solvent conditions, and was subject to energy minimization. After energy minimization, $\mathrm{MD}$ was performed at $300 \mathrm{~K}$ for 1,000 psec with 1 fsec each step.

Homodimerization. In order to obtain a homodimer, the same enzyme was used as the ligand and the protein. Like docking a ligand and protein assembly, a grid was prepared surrounding the protein. After setup the grid, another protein monomer considered as a ligand was docked. A protein and another protein assembly was subject to energy minimization. After energy minimization, MD was performed at $300 \mathrm{~K}$ for 500 psec with 1 fsec each step.

\section{Results and Discussion}

Three dimensional structures of OMT II-1 and OMT II-4. The 3D structure of OMT II-I obtained by homologybased modelling was compared with that of COMT. While COMT includes $20 \alpha$-helices and $9 \beta$-sheets, OMT II-I has 16 and 12, respectively. However, since the superimposition of backbones of two proteins gives RMSD value of $0.9 \AA$, it can be considered that they have a high similarity.

The structure of OMT ll-I was evaluated using PROC.HECK. Among 303 residues except Gly and Pro only one residue, Thr346 was found in disallowed regions of Ramachandran plot. The statistical analysis of Ramachandran plot showed that $89.4 \%$ are in the most favored regions, $8.9 \%$ in 
Table 1. A comparison of $\alpha$-helices and $\beta$-sheets included in OMT II-1, OMT II-4, and COMT (top: $\alpha$-helices; bottom: $\beta$-shets)

\begin{tabular}{|c|c|c|}
\hline OMT II-I & OMT II-4 & COMT \\
\hline Ast16 - Glul7 & Asno - Glu17 & \\
\hline Tyr21-Ser31 & Cys21-Ser31 & Glul 8 - Ser28 \\
\hline Pro3t - Ala40 & Pro34 - Ala40 & Ser 30 - Lell 42 \\
\hline Ile 49 - Lys 54 & Ile49 - Lys54 & $\begin{array}{l}\text { Asp } 43-\text { Ala51 } \\
\text { Ser58 - Ser64 }\end{array}$ \\
\hline Ile63 - Thr76 & Ile63 - Thr76 & Asp72- Tyr87 \\
\hline Aspl00-Argl05 & Aspl00-Arg105 & Thrl10-Vall 6 \\
\hline Phell5 - Aspl2l & Phel15-Aspl2l & \\
\hline Serl24 - Lell130 & Serl24 - Leul30 & Ile $124-G \ln 132$ \\
\hline Lys135 - Glul39 & Lys 135-Glu139 & $\begin{array}{l}\text { Asp 133-Glul38 } \\
\text { Serl39- Tyrl41 }\end{array}$ \\
\hline Hisl 43 - Val 148 & His 143 - Val 148 & Hisl42 - Gly 150 \\
\hline Gly 152 - Phel55 & Gly 152 - Phe 155 & Ile $152-$ Tyr 158 \\
\hline Gly 160 - His 184 & Gly 160 - His 184 & $\begin{array}{l}\text { Thrl61 - Hisl } 66 \\
\text { Aspl69- Tyr } 195\end{array}$ \\
\hline Ser $204-G 1 y 211$ & Ser204-Gly211 & $\begin{array}{l}\text { Gly212 - Thr217 } \\
\text { Ile218 - Tyr222 } \\
\text { Lel1232 - Asp238 }\end{array}$ \\
\hline Ile $263-\mathrm{His} 276$ & Ile263- His 276 & $\begin{array}{l}\text { Ile } 267 \text { - Trp } 271 \\
\text { Ser272 - Lelı } 287\end{array}$ \\
\hline Glu298 - Thr313 & Glu298 - Thr313 & Ser307 - Asn324 \\
\hline Leu322 - Thr 332 & Leu322 - Thr 332 & Gln332 Ala 342 \\
\hline OMT II-I & OMT II-4 & COMT \\
\hline Leu46 - Glu47 & Len46 - Glu47 & \\
\hline Arg $80-A \operatorname{la} 86$ & Arg 80 - Ala86 & \\
\hline Thr92 - Leu98 & Thr92 - Leu98 & $\begin{array}{l}\text { Leu90 - Thr96 } \\
\text { Val102 - Leul08 }\end{array}$ \\
\hline Leu193 - Lys197 & Leu193 - Lys197 & Leli204 - Val207 \\
\hline Val216-Thr221 & Val216 - Thr221 & \\
\hline His 235 - Asp239 & His $235-$ Asp239 & Gly 227 - Asp231 \\
\hline Asp252 - Met253 & Asp252-Met 253 & Val245 - Gly 249 \\
\hline Asin 283 - Ser 287 & Asn283-Ser287 & Val262 - Phe 263 \\
\hline Pro289 - Ala290 & Pro289 - Ala290 & Lys292 - Leu300 \\
\hline Lle $320-\operatorname{Met} 321$ & Ile $320-$ Met321 & \\
\hline Lys337 - Leu339 & Lys337 - Leu339 & Val349-Ala353 \\
\hline Gly347 - Val351 & Gly347 - Val351 & Thr356 - Leu362 \\
\hline
\end{tabular}

additional allowed regions. and $1.3 \%$ in generously allowed regions. G-factor is 0.11 .

OMT II and substrate assembly. The substrate. caffeic acid and cofactor. SAM binding sites of OMT II-l and OMT II-4 were determined based on a comparison of the corresponding residues with binding sites of COMT (Table 2). In the case of the substrate binding site. Ile3 16 of COMT is switched with Leu317 in OMT II-I and OMT II-4. However in the case of the SAM binding site. all residues are agreed with each other. Even though OMT IIs form a substrate assembly a comparison of OMT II-l with OMT II-4 shows that there is no difference in both the substrate and the cofactor binding sites. Therefore, the different binding affinity over caffeic acid of OMT II-I and OMT II-4 cannot be explained by their primary sequences composing the binding sites.

The quatemary structure of OMT II-1 and OMT II-4. In order to explain the binding difference in OMT II-l and OMT II-4 in other way, we turn our attention to the quatemary structure of these proteins. The homodimer of OMT II-l was obtained by docking OMT II-I into another OMT II-I (Figure 2). The N-tenminals from each subunit lie in the opposite direction as found in COMT. It was known that COMT consists of two polymers $A$ and $C$ which are dimerized by contact of the N-terminal domain. To compare OMTII-I homodimer with COMT homodimer. they are superimposed and its RMSD value is $8.1 \AA$ (Figure 3 ). A comparison of OMTII-I monomer with COMT monomer gives the RMSD value of $7.3 \AA$. That is. the case of dimmer shows better RMSD than that of monomer. As shown in Table 1, the N-terminal domain of COMT includes three helices between Glul8 and Ala5l. However. OMT II-1

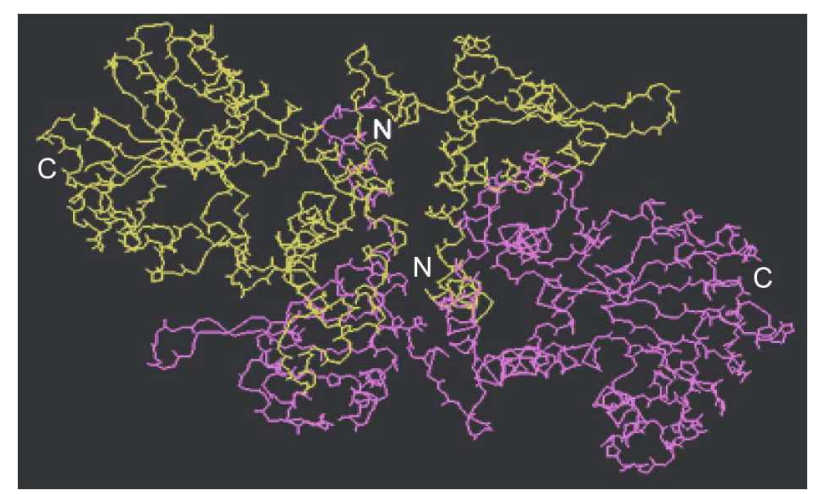

Figure 2. The structure of OMT II-1 homodimer. (white, a monomer; grey, another monomer)

Table 2. A comparison of residues neighboring binding sites of OMT II-1, OMT II-4, and COMT

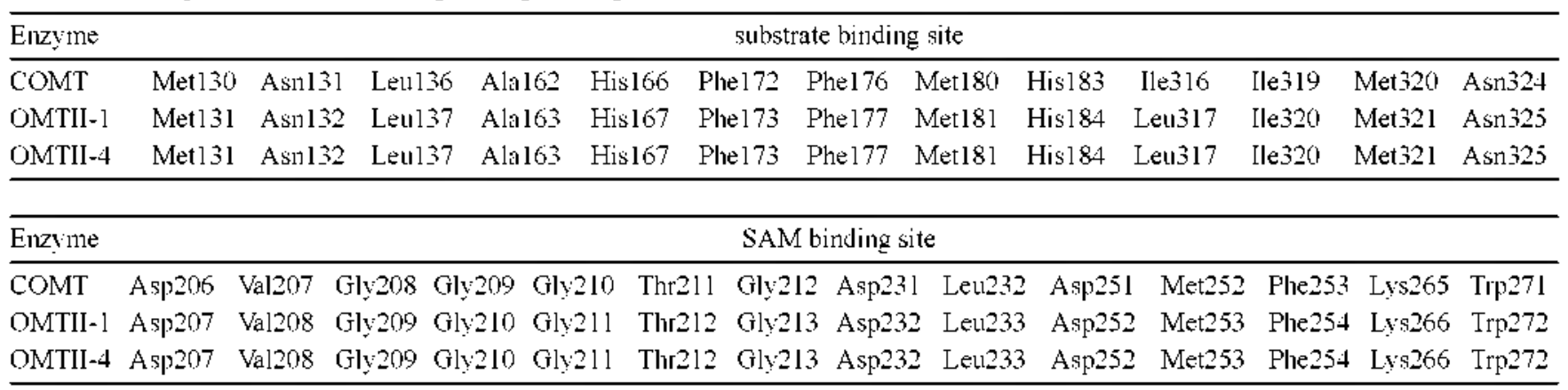




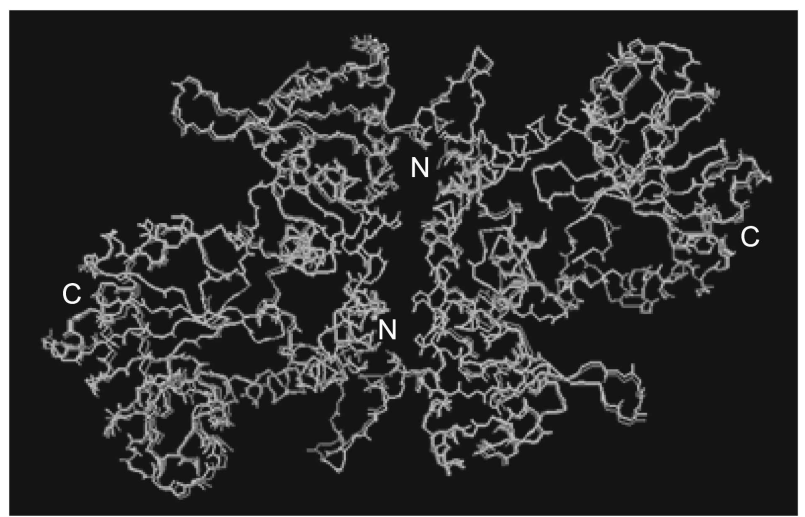

Figure 3. A superimposition of OMTII-I homodimer (white) on COMI homodimer (grey).

appears to have one more helix that is composed of twelve amino acids between Asn 6 and Glu17. Since the helix structure is not flexible, the presence of one more helix in OMT II-1 can make wider contact area and it could facilitate dimer formation. The driving forces for dimerization are electrostatic interactions and van der Waals interactions. As listed in Table I, having one more helix in OMT [l-1 results in more van der Waals force for dimer formation. Comparing the residues showing van der Waasls interaction, included in opposing $\alpha$-helices of each N-terminal, OMT II-I has nine more residues than COMT. It would confer more van der Waals force when OMT II-I forms dimer than when COMT does. In addition, while there are six continuing Glu residues in the first helix of OMT [l-1, there are only two Glu residues in COMT. As a result, it may cause stronger electrostatic interactions. Taken together, OMT $11-1$ is predicted to form dimer." Two OMT cDNA clones were isolated from freshwater weed Chrysosplenim americamm. ${ }^{15}$ They have only three residue difference but they show a large substrate specificity. While one OMT (NCBI Accession No.: P59049) shows $100 \%$ binding affinity for quercetin. another OMT (NCBl Accession No.: Q42653) does 38\%. Of course, three residues are included in the binding sites. Therefore, this phenomenon can be explained based on the dimmer interface interaction.

OMT II-4 homodimer was also obtained by the same method as applied on OMT [I-1. As shown in Figure I, Tyr 21 of OMT Il-1 is switched with Cys 21 in OMT 1$]-4$. However, the comparison of helices of OMT II-I with those of OMT 11-4 shows that there is no difference (Table 1). While OMT [I-] homodimer showed the binding affinity of $100 \%$ over caffeic acid. OMT $11-4$ homodimer did $25 \%$." Since OMT IIs are predicted to form dimer, the quaternary structures of OMT Ils cause the substrate binding differences.

The characteristic in the dimer formation of COMT is that Ser28 sticks out into the binding site of the other monomer. ${ }^{16}$ At the same time, the residues being close to Ser 28 move closer to the binding site of the other monomer. It helps to exclude solvent in the substrate binding pocket. In the cases of OMT IIs, Ser29 matches Ser28 of COMT. The second helix of OMT IIs is composed of twelve residues between
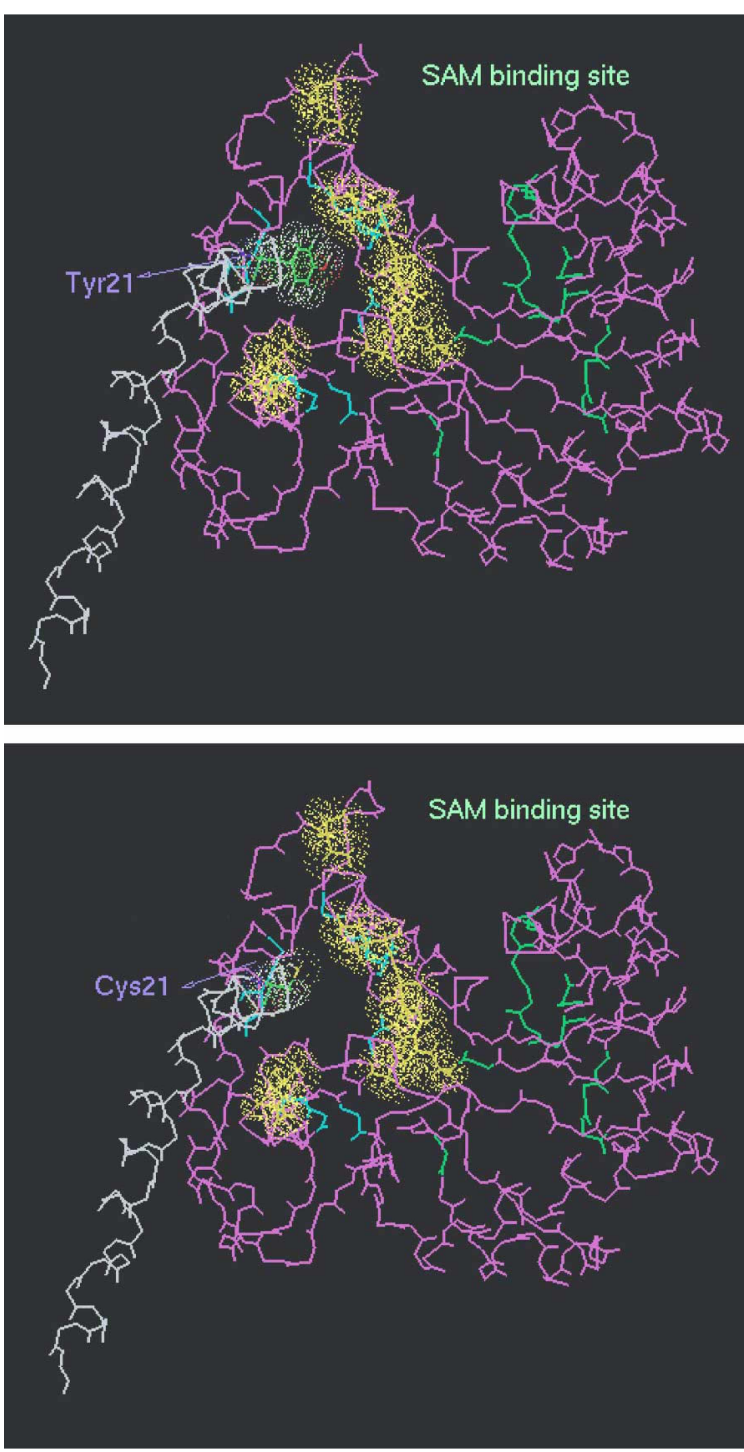

Figure 4. A comarison of the V-terminal domain of OMT II-1 homodimer backbone (top) with that of OMT II-4 homodimer backbone (bollom). (I)ols denole the residues showing wan der Waals interaction with lyy 21 or (ys2I.)

the 21 st residue and the 31 st. Tyr2l and Cys 21 are the starting residue of the helix. Therefore, the residues being close to Ser29 might move closer to the substrate binding pocket as those of COMT. To clarify this conjecture, the residues showing van der Waals interaction with Tyr $2 \mathrm{l}$ of OMT 11-1 were investigated in the other monomer. Eight residues of the other monomer were found. which were Phe1 15, Met125, His184, Jlel87, Thrl88, Thr313, Phe355, and Gly356. Unlike OMT II-], only three residues were observed in Cys2] of OMT II-4, which were Phell5, Met125, and Phe355. This phenomenon can be explained by the reason why Tyr2l being closer to the binding site of the other monomer can help to keep out solvent molecules from the binding site and make the substrate access the binding site easier. As a result, the shorter distance between Tyr2I and the binding site of the other monomer probably causes the better binding aftinity. 
Acknowledgement. This work was supported by Frontier 21 Crop Functional Genomics Center. Korea Ministry of Science and Technology (CG2214).

\section{References}

1. Dixon. R. A. Nature 2001. H1, 843 .

2. Maury. S.: Geotiroy. P.: Legrand. M. Plam Phisiol 1999. 121. 215.

3. Zhong, R.: Morrison. W. H. III: Himmelsbach. D. S. Poole, F. L.: Ye Z. H. Plant Phnsiol. 2000, 124. 563

4. Schaller, H. Bouvier-Navé. P.: Benteniste, P. Plant Plysiol. 1998. $118,461$.

5. Wang. J.: Pichersky. E. Arch. Biochem. Bioply. 1999.368. 172.

6. Ibrahim. R. K.: Bruneau. A.: Bantignies. B. Plant Mol. Biol. 1998. 36.1 .
7. Muzac. I: Wang, J: Anzellotti, D.: Zhang, H.: Ibrahim. R. K. Arch. Biochem. Biophy 2000. 375. 385.

8. Echardt. N. A. Plam Cell. 2002. J4. 1185.

9. Moon. J. K.: Kim. J.: Rhee. S.: Kim. G.: Yut1. H.: Chung. B.: Lee. S.; Lim. Y. Bull Korean Chent. Soc. 2002, 23. 1545.

10. Choe. J.: Chang, S. Bull Korean Chent. Soc. 2002, 23, 48.

11. Frick. S.: Kutchan. T. M. Plant J. 1999, 17,329.

12. Wilmouth. R.: Turnbull. T.: Welford. R.: Clifton. I.: Prescott. A.: Schofield. C. The Protein Data Bank. Structure (London 2002. 10.93 .

13. Van Aalten. D. M. F; Crielaard. W: Hellingwerf. K. J.: JoshuaTor, L. Acta Crystallogr: Sect. D 2002. 58.585.

14. Yoon, E. Y. Bull. Kowam Chem Soc. 2001. 22. 293.

15. Gauthier. A.; Gulick. P. J.; Ibrahim. R. K. Arch. Biochem. Biophy? 1998.351 .243

16. Zubieta. C.: Kota. P.: Ferrer. J.: Dixon. R. A.: Noel. J. P. PIant Cell 2002. Jf. 1265 . 